various types of composite structures such as pressure vessels, pipes, domes, shafts and tanks, to name but a few, introduced. Appendices have been revised too and now include SCILAB code for Classical Lamination Theory as well as details of Periodic Microstructure Micromechanics and Longitudinal Compressive Strength.

I have no doubt that the Third edition of this textbook will help the new generation of readers to gain a better understanding of material selection, fabrication, material behaviour and structural analysis involved in design of composite structures.

Professor Maria Kashtalyan School of Engineering, University of Aberdeen, Aberdeen, UK

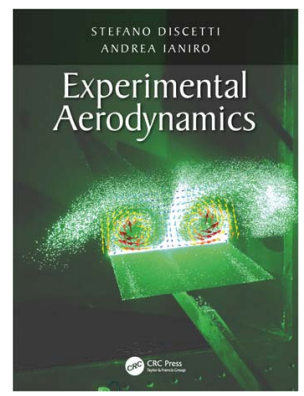

\section{Experimental Aerodynamics}

\section{Edited by S. Discetti and A. Ianiro}

CRC Press, Taylor \& Francis Group, 6000 Broken Sound Parkway NW, Suite 300, Boca Raton, FL, 33487-2742, USA. 2017.

Distributed by Taylor \& Francis Group, 2 Park Square, Milton Park, Abingdon, OX14 4RN, UK. xiii; 468pp. Illustrated. £120. (20\% discount available to RAeS members via www.crcpress.com using AKQ07 promotion code). ISBN 978-1-4987-0401-4.

7 here have very few text or reference books on experimental aerodynamics. Previous works have focused on wind-tunnel testing or provide a general overview of different experimental techniques with some limited applications. This book attempts to cover this gap in the market by bringing together different contributors from around the world to in compiling a one-stop reference/textbook for students and practitioners in aerospace engineering.

Overall, the book is focused on aerodynamic applications and methods are presented with this in mind. The entire range of 
Mach numbers from low-speed wind tunnels to super and hypersonic wind tunnel tests are considered. The book is divided in four sections. The first section covers the basic aspects of experimental aerodynamics. This includes the theory (Chapter 1), types of facilities (Chapter 2) as well as the principles of flow visualisation (Chapter 4). Each of these chapters is written by experts with experience as well as exposure to a broad range of techniques.

One of the most important chapters of Section I is Chapter 3 which delves in to statistical data analysis. This is very crucial and is an important chapter as it pertains to the different types of analysis that can be performed with the range of data that can acquired with measurement techniques. It is very nice to see the inclusion of modal decomposition techniques that is becoming widely used with imaging data. This chapter also addresses the issues related to uncertainty analysis (a topic that is often ignored in technical publications). Some of these chapters in Section I are introductory in nature and depending on the background/ level of the students/practitioners these can be selectively skipped.

The second section is concerned with scalar measurements and this consists of four chapters (5-8) and covers techniques for pressure (Chapter 5), temperature/heat-flux (Chapter 6), density (Chapter 7) and interferometry/holography (Chapter 8) measurements. The four different chapters are written by four different individuals with experience and background in those methods. It is a little disappointing to see that spectroscopic measurements is not included in this section, primarily because these methods are starting to be used in high-speed facilities (supersonic/hypersonic speeds). This book could have touched upon these methods and referred to other papers. This way, readers could have a gateway to look in to those more advanced techniques. However, I also realise the difficulties associated with drawing a line. Given that constraint, I believe these chapters cover the essentials of scalar measurements.

The third section deals with velocity measurements and covers thermal anemometry (Chapter 9), laser anemometry (Chapter 10) and volumetric measurements (Chapter 11). These three chapters cover the range the entire range of velocity measurement techniques. The level of detail is varied in each chapter; however, it does provide a broad overview of the methods. Perhaps, one technique that is missing is light-field imaging as this is a new development for imaging 3D volumes. However, this might be regarded as a minor detail.

The final section details with wall-shearstress and force measurements. Most common shear-stress measurement systems are reviewed, and pros and cons of each method is presented. Micro-electro-mechanical systems (MEMS)-based shear-stress sensors are not covered in any depth. However, there are several reference works and textbooks that cover this material and perhaps the authors felt that it is not necessary to do this here. The force measurements chapters introduce different types force/moment balances; however, none of these are covered in any depth. I am little puzzled that this section appears at the end of the book as force/ moment measurements are perhaps the first thing practitioners would be interested in the 
most. Overall, all the necessary information about these types of measurements is here.

In conclusion, this book is intended to expose aerodynamicists to different measurement methods and provide a gateway for individuals to further develop their interest. A unique aspect of this book is the problem sheet at the end of each chapter and this would certainly allow users to test themselves. This type of content is usually not available in experimental techniques books and usually users have to carry out the experiment to demonstrate their understanding of the methods. However, this unique aspect allows this book to be accessible to non-experimentalists and, therefore, could have a wider reach. If this was the intention, then I applaud the authors for editing a good book that has something in it for all aerodynamicists.

Professor Bharathram Ganapathisubramani, FRAeS Professsor of Experimental Fluid Mechanics

University of Southampton, Southampton, UK

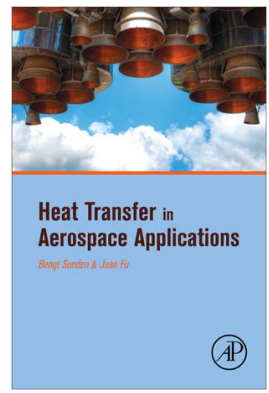

\section{Heat Transfer in Aerospace Applications}

\section{B. Sunden and J. Fu}

Academic Press, Elsevier, The Boulevard, Langford Lane, Kidlington, Oxford, OX5 1GB, UK. 2017. xv; 255pp. Illustrated. £104. ISBN 978-0-12-809760-1.

T was quite excited about the book as there has not been any book on heat transfer especially dedicated for the aerospace applications. The heat transfer concept has been explored and explained in many ways for various engineering applications but this first edition book describes the concept of heat transfer in the context of aerospace applications. The aerospace environment itself poses various challenges for aircraft/spacecraft such as microgravity, extreme temperatures and pressures, aerodynamic heating, vibrations, shock loads and extended duration of operations. This book consists of 11 different chapters explaining the heat transfer concepts/problems for these various aerospace challenges.

The first chapter introduces the heat transfer concept in general and summarises the specifics for aerospace heat transfer. 